$05.1 ; 06.4 ; 13.2$

\title{
Аномальные характеристики ионной эмиссии при разрыве полимеров
}

\author{
(C) В.А. Пахотин, Ю.М. Бойко \\ Физико-технический институт им. А.Ф. Иофффе РАН, Санкт-Петербург, Россия \\ E-mail: v.pakhotin@mail.ioffe.ru
}

Поступило в Редакцию 1 сентября 2020 г.

В окончательной редакции 8 октября 2020 г.

Принято к публикации 9 октября 2020 г.

\begin{abstract}
Проведены измерения вольт-амперных характеристик эмиссии ионов при разрыве пленочных образцов полиэтилентерефталата, полиимида и полифениленэфира в высоком вакууме. Положительные ионы начинают обнаруживаться при тянущем потенциале $100-200 \mathrm{~V}$, и их число монотонно увеличивается до насыщения с ростом потенциала. Эмиссия отрицательных ионов наблюдается при потенциале $200 \mathrm{~V}$ и в диапазоне 0-50 V имеет резонансный характер, причем положение резонансных пиков определяется природой полимера. Обнаруженный резонансный характер эмиссии отрицательных ионов соответствует предлагаемому физическому механизму образования ионов.
\end{abstract}

Ключевые слова: полимерные пленки, ионная эмиссия, микротрещина.

DOI: 10.21883/PJTF.2021.02.50545.18535

Связь механических и электрофизических эффектов в твердых телах привлекает внимание вследствие большой научной и практической значимости. Одно из явлений, возникающих при механических воздействиях на твердые тела, - эмиссия заряженных частиц (механоэмиссия) [1-3]. С появлением быстродействующей регистрирующей цифровой техники удалось обнаружить эмиссию ионов при разрушении широко распространенных полимеров [4]. В течение примерно одной миллисекунды (время разрушения) испускаются как отрицательные, так и положительные ионы. Установлено, что кинетика эмиссии зависит от физических свойств исследуемого полимера. С помощью специальных опытов было показано, что эмиссия ионов возникает при движении магистральной трещины, проведен расчет траекторий движения ионов в трещине [4,5]. Установлено, что в эксперименте преимушественно должны регистрироваться ионы, вылетевшие с поверхности образца в вершине растущей трещины. Ширина области эмиссии составляет $\delta \sim 20 \mu \mathrm{m}$ (рис. $1, a$ ). Эмиссия положительных ионов также обнаружена в процессе разрушения адгезионных соединений полимеров [6,7]. Как показали исследования, эмиссия ионов возникает при деформировании и разрушении различных полимеров. Таким образом, это общее явление. Оно обусловлено, с одной стороны, механическими свойствами материала, и поэтому исследование эмиссии позволит детализировать картину механического разрушения. С другой стороны, эмиссия заряженных частиц - это электрофизический процесс, обусловленный свойствами полимера, точнее, свойствами макромолекул, особенностями воздействия механических напряжений на межатомные химические связи и релаксацией упругих напряжений при разрывах макромолекул. Исследование этого явления важно как для физики прочности полимеров, так и для разработки различных датчиков физических полей [8]. Однако явление механоэмиссии исследовано далеко не полностью; в частности, не измерены вольт-амперные характеристики $(\mathrm{BAX})$ эмиссии.

В настоящей работе исследована зависимость числа эмитированных ионов $N$ от величины тянущего электрического потенциала $U$ (BAX). Схема эксперимента показана на рис. 1,a. Объектами исследования были широко используемые в промышленности полимеры: полиэтилентерефталат (РЕТ), полиимид типа ПМ-1 (PI) и полифениленэфир (poly(2,6-dimethyl-p-phenylene ether)) (PРЕ). Пленки РЕТ имели толщину $30 \mu \mathrm{m}, \mathrm{PI}-40 \mu \mathrm{m}$, $\mathrm{PPE}-100-150 \mu \mathrm{m}$. Образцы в форме двусторонней лопатки устанавливались в вакуумной камере с помощью металлических зажимов. Ширина рабочей части образцов составляла $4 \mathrm{~mm}$. Длина рабочей части образцов составляла $5 \mathrm{~mm}$. Деформирование образцов проводилось при комнатной температуре с постоянной скоростью $3.78 \mathrm{~mm} / \mathrm{s}$. Образцы не имели надреза. Растяжение образцов осуществлялось в камере, изготовленной из нержавеющей стали, при комнатной температуре [4]. Давление остаточных газов в камере составляло $\sim 10^{-5} \mathrm{~Pa}$.

Измерение интенсивности эмиссии проводилось в режиме счета частиц с использованием вторичноэлектронного умножителя ВЭУ-6 (СЕМ) с заземленным катодом. Умножитель был расположен на расстоянии $L=0.28$ m от образца с целью обеспечения возможности создания магнитного поля между сеткой и детектором частиц. При измерении механоэмиссии выделение ионной компоненты в потоке отрицательно заряженных частиц осуществлялось путем отклонения электронов поперечным магнитным полем, создаваемым двумя постоянными магнитами плоской формы. В центральной 
области между магнитами индукция поля составляла 0.036 T. Для обеспечения отбора эмиссионного тока частицы ускорялись в промежутке между изолированным электродом и заземленной сеткой, установленной на входе дрейфового пространства (пролетной трубы). BAX записывались при подаче на электрод потенциала $U$ в диапазоне $0-1000 \mathrm{~V}$, знак которого совпадал со знаком заряда регистрируемых частиц. Расстояние между электродом и сеткой составляло $l=22 \mathrm{~mm}$. Измерение интенсивности эмиссии осуществлялось синхронно с записью диаграммы нагружения образцов.

Как было показано раньше [4], эмиссионный всплеск ионов любого знака при разрыве имеет длительность $\leqslant 1 \mathrm{~ms}$. Пока измерительная система не позволяет осуществлять развертку напряжения на электроде и синхронную регистрацию эмиссии в течение столь короткого времени. Поэтому образцы деформировались при фиксированных потенциалах на электроде. В отличие от работы [4], где счет импульсов осуществлялся каждую миллисекунду и только во время разрыва каждую микросекунду, система производила подсчет числа импульсов детектора за каждую микросекунду в ходе всего эксперимента. На интерфейс системы можно было выводить из памяти компьютера полученные сигналы как в микросекундном масштабе, так и в миллисекундном масштабе времени.

Измерения показали, что ВАХ положительных ионов для всех исследуемых полимеров имеют примерно один и тот же вид (рис. $1, b)$ : заметная эмиссия начинается c $200 \mathrm{~V}$ и ее интенсивность постепенно увеличивается до насыщения с увеличением тянущего потенциала. Это обусловлено свойствами детектора частиц (СЕМ) [9], который эффективно регистрирует положительные ионы достаточно высоких энергий. Эмиссия отрицательных ионов имеет другой характер: возникает при относительно низких напряжениях и, что особенно интересно, в этой области напряжений наблюдаются заметные выбросы на кривых эмиссии (рис. 2). В случае PI наибольшая эмиссия наблюдается при $45 \mathrm{~V}$, в случае PET - при $50 \mathrm{~V}$, случае PРE - при $16 \mathrm{~V}$. Тот факт, что эмиссия практически отсутствует при тянущем напряжении между электродом и сеткой, равном нулю, свидетельствует о том, что наблюдается эмиссия ионов, а не нейтральных частиц. Кроме того, нейтральные частицы для их регистрации должны быть предварительно ионизованы [10]. Важным обстоятельством является чувствительность положения резонансных пиков эмиссии к типу полимера. Полученные результаты соответствуют современным представлениям об образовании отрицательных ионов путем резонансного захвата электронов нейтральными молекулами. Нейтральные фрагменты макромолекул образуются в процессе разрушения полимерного образца. Энергетические сечения захвата электрона у разных молекул заметно различаются, поэтому для фрагментов макромолекул различных полимеров требуется разная энергия электронов. Молекулы присоединяют избыточный электрон низкой энергии -

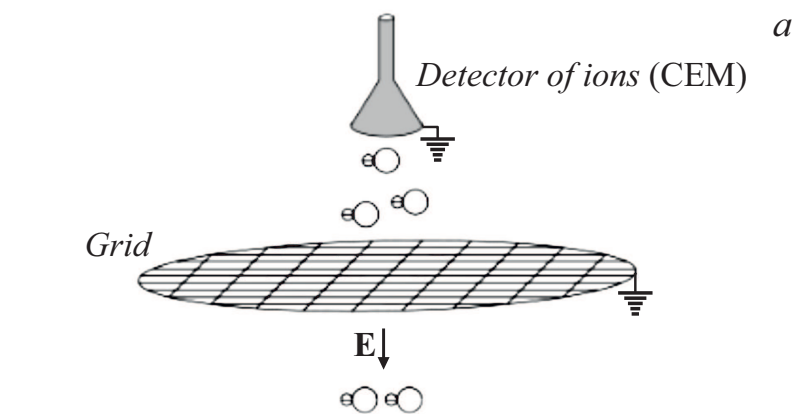

$a$

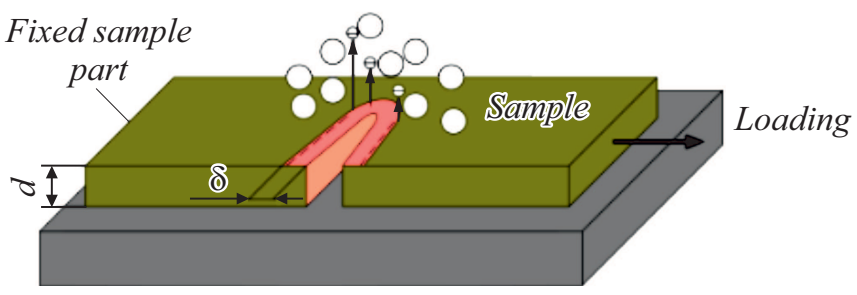

Electrode $U, \mathrm{~V}$

$b$

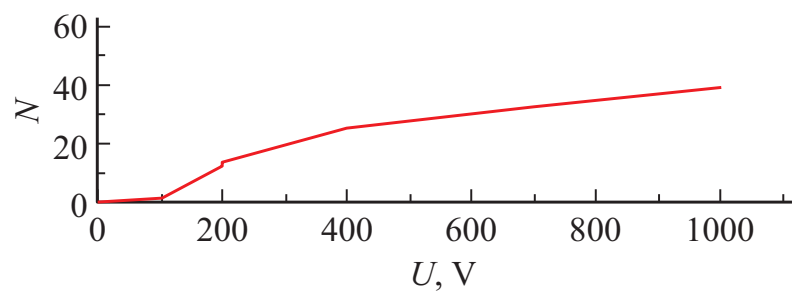

Рис. 1. $a-$ схема измерительной установки и образования отрицательных ионов при прорастании магистральной трещины в образце разрываемого полимера. Большие кружки - нейтральные молекулы, маленькие кружки - электроны. $b$ - зависимость числа эмитированных положительных ионов от тянущего потенциала при разрыве образца полиимида.

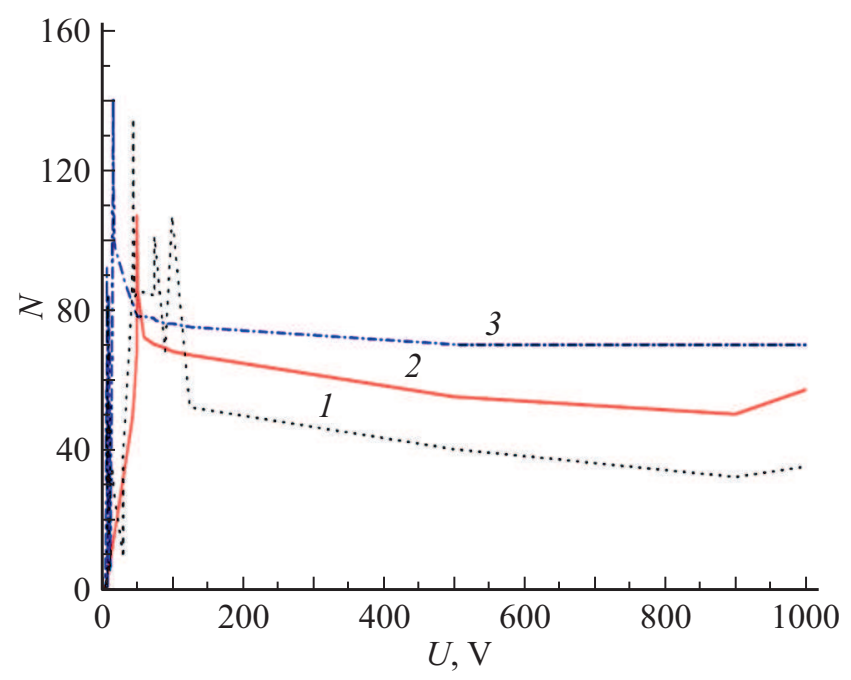

Рис. 2. Зависимость числа эмитированных отрицательных ионов от тянущего потенциала при разрыве образцов полимеров. 1 - PI, 2 - PET, 3 - PPE.

от тепловой до $10-15 \mathrm{eV}$ [11]. В наших опытах резонансные пики наблюдаются при относительно больших 
тянущих потенциалах (до десятков вольт). Это объясняется тем, что концентрация летучих нейтральных продуктов велика вблизи поверхности, что и определяет наибольшую вероятность захвата. Поэтому электрон не успевает набрать энергию, сравнимую с поданным на электрод потенциалом. Можно оценить вероятность захвата эмитированного электрона и показать, что она имеет максимум при некотором потенциале электрода $U_{m}$. Вероятность прилипания электрона к молекуле определяется выражением [12]:

$$
P=\sigma v_{p} n t_{p}
$$

где $\sigma$ - сечение прилипания, $v_{p}-$ скорость электрона в момент прилипания, $t_{p}$ - время движения электрона, $n$ - концентрация молекул. При помещении образца диэлектрика в электрическое поле возникает три зоны (в нашем случае две) с электрическим полем. Первая в диэлектрике, вторая - вне диэлектрика. Образец полимера прижат без зазора одной стороной к электроду. При потенциале электрода, равном $U$, напряженность поля вне образца (в вакууме) $E_{v}$ будет составлять

$$
E_{v}=\frac{U k}{d} \varepsilon
$$

где $\varepsilon$ - относительная диэлектрическая проницаемость образца. Коэффициент $k$ учитывает поляризацию образца:

$$
k=\frac{1}{1+\frac{\varepsilon \Delta l}{d}} .
$$

Здесь $d-$ толщина образца, $l$ - расстояние между электродом и сеткой, $\Delta l-$ расстояние между поверхностью образца и сеткой: $\Delta l=l-d$. Поле $E_{v}$ превышает поле в диэлектрике (образце) в $\varepsilon$ раз. Электроны, эмитируемые с поверхности образца, попадают в поле $E_{v}$. Поэтому наблюдаемые эффекты определяет именно поле в вакууме. Оно и использовалось в расчетах. Чтобы ослабить влияние образца, следует $k$ уменьшать. Самый простой путь - увеличить расстояние между электродом и сеткой так, чтобы $\Delta l \gg d$. В наших экспериментах так и было сделано.

При наличии тянущего поля траектории электронов будут приблизительно лежать на нормали к поверхности образца. Будем считать вылет молекул изотропным. По мере удаления от поверхности полимера их концентрация будет уменьшаться квадратично с расстоянием

$$
n=n_{0}\left[\frac{1}{1+\left(\frac{x}{d_{0}}\right)^{2}}\right]
$$

Здесь $n_{0}$ - начальная концентрация молекул, $x$ расстояние от поверхности образца, $d_{0}-$ расстояние, на котором концентрация уменьшается в 2 раза. Введем следующие обозначения: $W_{p}$ - энергия прилипания, $W_{0}$ - начальная (тепловая) энергия электрона, $q-$ заряд электрона.

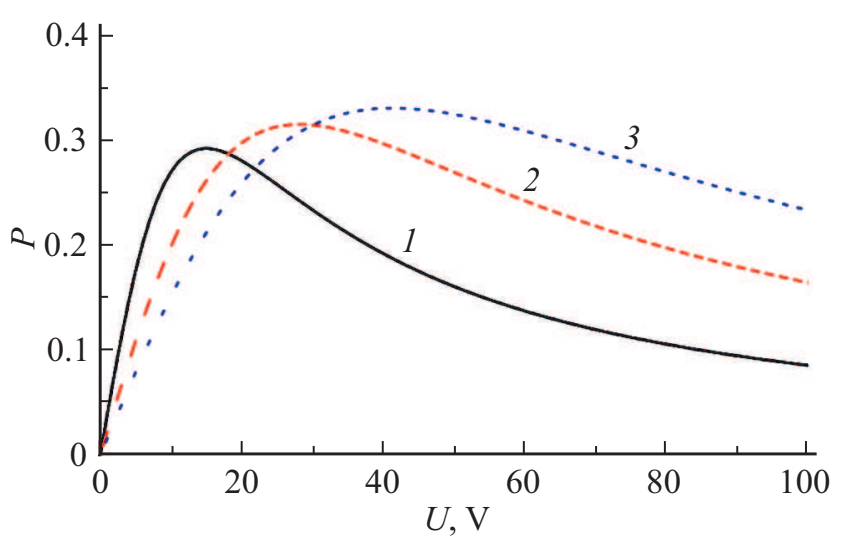

Рис. 3. Зависимость вероятности образования отрицательного иона от потенциала электрода при $W_{p}=0.12(1), 0.18$ (2) и $0.24 \mathrm{eV}(3)$.

Далее, выразив в (1) и (4) $t_{p}$ и $x$ через тянущий потенциал $U$, найдем вероятность прилипания как функцию $P(U)$. Эти выкладки относительно громоздки и поэтому не приводятся. Зависимости $P(U)$ для различных $W_{p}$ приведены на рис. 3. Максимум на кривых находится при потенциале

$$
U_{m}=\frac{\Delta l\left(W_{p}-W_{0}\right)}{q d_{0}(1-k)} .
$$

Энергии $W_{p}=0.12 \mathrm{eV}$ соответствует $U_{m}=14.98 \mathrm{~V}$, $W_{p}=0.18 \mathrm{eV}$ отвечает $U_{m}=28.14 \mathrm{~V}, W_{p}=0.24 \mathrm{eV}$ соответствует $U_{m}=41.3 \mathrm{~V}$. Оценки сделаны для $\varepsilon=3$, $d_{0}=100 \mu \mathrm{m}, T=600 \mathrm{~K}$ (начало процесса термодеструкции РЕТ), $\sigma=6 \cdot 10^{-20} \mathrm{~m}^{2}, n_{0}=8 \cdot 10^{22} \mathrm{~m}^{-3}$.

Если подставить в формулу для напряженности поля в вакууме (2) выражение для $k(3)$ и потенциал $U_{m}$, то получим формулу для напряженности поля, соответствующей наибольшей вероятности прилипания:

$$
E_{m}=\frac{W_{p}-W_{0}}{q d_{0}} .
$$

Из формулы (6) следует, что напряженность поля $E_{m}$ является инвариантом по отношению к изменению межэлектродного расстояния. Если на опыте так и окажется, то это будет подтверждением предложенного механизма образования анионов. Это тема для дальнейшей работы.

Небольшая величина энергии захвата свидетельствует о том, что электрон прилипает к сложным молекулам, каковыми могут являться фрагменты разорванных макромолекул полимера. При этом времена жизни отрицательных ионов, образованных в результате прилипания электронов к соответствующим макромолекулам, определяются числом колебательных степеней свободы этих молекул и могут составлять десятки микросекунд [13]. Поэтому значительная часть отрицательных ионов, вылетающих при распространении трещины, достигает детектора частиц. Прилипание электронов к молекулам может происходить и в конденсированной фазе. Этот 
процесс в объеме полимера облегчен в силу увеличения возможных путей передачи энергии, обусловленных взаимодействием молекул. Кроме того, сечение прилипания электрона возрастает при возбуждении макромолекулы. Следовательно, локальное и значительное повышение температуры при разрушении полимера и связанное с этим колебательное возбуждение молекул ведут к росту вероятности прилипания электрона. В связи с этим можно предположить, что ток отрицательных ионов, регистрируемый при потенциалах электрода выше $100 \mathrm{~V}$, состоит из макроионов, образованных в конденсированной фазе, поскольку при таких энергиях электроны могут оказаться нерезонансными.

Таким образом, в работе экспериментально показано, что при небольших тянущих напряженностях электрического поля $(E<5 \mathrm{~V} / \mathrm{mm})$ ток механоэмиссии отрицательных ионов имеет резонансный характер, причем положение пиков определяется природой полимера. В качестве рабочей гипотезы принято, что подобный характер объясняется резонансным захватом электронов нейтральными летучими продуктами разрушения полимера. Сделанные оценки показывают, что вероятность прилипания электрона в вакууме, движущегося в условиях уменьшающейся концентрации молекул, имеет экстремальную зависимость от ускоряющего потенциала. Возможно образование фрагментов макромолекул с различными $W_{p}$ при разрыве одного и того же полимера. По-видимому, число резонансных пиков и их положение можно использовать для определения различных распределений фрагментов макромолекул. Дальнейшее исследование механоэмиссии ионов, в частности, с использованием методов масс-спектрометрии без ионизации позволит разработать физический механизм образования ионов и выхода их в вакуум, что даст возможность уточнить механизм разрушения полимеров под нагрузкой. Применение нового ионного источника, роль которого выполняет разрушаемый полимер, может дать возможность исследовать отрицательные ионы, которые практически невозможно создать с помощью ионных источников, используемых в масс-спектрометрии.

\section{Финансирование работы}

Работа выполнена при финансовой поддержке Российского фонда фундаментальных исследований (проект № 18-08-00401 A).

\section{Конфликт интересов}

Авторы заявляют, что у них нет конфликта интересов.
[3] J. Fuhrmann, G.H. Scherer, L. Nick, Makromol. Chem. Phys., 188, 2241 (1987). https://doi.org/10.1002/macp.1987.021880921

[4] В.А. Закревский, В.А. Пахотин, ФТТ, 52 (6), 1083 (2010). https://journals.ioffe.ru/articles/viewPDF/1857

[5] В.А. Пахотин, В.А. Закревский, Н.Т. Сударь, А.А. Апасеев, Науч.-техн. ведомости СПбГПУ. Физ.-мат. науки, 11 (2), 106 (2018). https://doi.org/10.18721/JPM.11210

[6] Yu.M. Boiko, V.A. Zakrevskii, V.A. Pakhotin, J. Adhesion, 90, 596 (2014). http://dx.doi.org/10.1080/00218464.2013.822305

[7] Yu.M. Boiko, V.A. Zakrevskii, V.A. Pakhotin, N.T. Sudar, Colloid Polym. Sci., 297, 831 (2019). https://doi.org/10.1007/s00396-019-04505-6

[8] В.А. Закревский, В.А. Пахотин, Радиотехника и электроника, 57 (1), 62 (2013). https://doi.org/10.7868/S0033849412100129

[9] J.H. Gross, Mass spectrometry (Springer, Berlin-Heidelberg, 2004). https://doi.org/10.1007/3-540-36756-X

[10] М.Р. Айнбунд, Б.В. Поленов, Вторично-электронные умножители открытого типа и их применение (Энергоатомиздат, М., 1981).

[11] В.А. Мазунов, П.В. Щукин, Р.В. Хатымов, М.В. Муфтахов, Масс-спектрометрия, 3 (1), 11 (2006).

[12] H. Massey, Negative ions (Cambridge University Press, 1976). [Г.С.У. Месси, Отрицательные ионы (Мир, М., 1979).].

[13] Е. Илленбергер, Б.М. Смирнов, УФН, 168 (7), 731 (1998). https://doi.org/10.3367/UFNr.0168.199807c.0731

\section{Список литературы}

[1] В.А. Закревский, В.А. Пахотин, ФТТ, 20 (2), 371 (1978).

[2] J.T. Dickinson, E.E. Donaldson, M.K. Park, J. Mater. Sci., 16, 2897 (1981). https://doi.org/10.1007/BF00552976 